\title{
Low complexity cyclic autocorrelation function computation for spectrum sensing
}

\author{
Shusuke Narieda ${ }^{\text {a) }}$ \\ Dept. of Electr. and Comput. Eng., National Institute of Technology, Akashi College, \\ 679-3 Nishioka, Uozumicho, Akashi, Hyogo 674-8501, Japan \\ a)narieda@akashi.ac.jp
}

Abstract: This letter presents a low complexity cyclic autocorrelation function (CAF) computation technique for the spectrum sensing of orthogonal frequency division multiplexing (OFDM) signals in cognitive radio. Although CAFs are frequently employed in signal cyclostationarity detection (CD) based spectrum sensing, the computational complexity of CAFs is greater than that of signal energy. The presented technique computes the CAF by multiplying a received signal, delayed one and complex periodic function where these signals and function are thinned-out so that the sampling frequency becomes quadruple of a cyclic frequency. The employed function can only take the values \pm 1 and 0 . Because the multiplication and addition can omit in the CAF computation, the computational complexity can be reduced. We numerically verify the effectiveness of the presented CAF computation technique.

Keywords: cyclic autocorrelation function, cognitive radio, cyclostationarity detection based spectrum sensing

Classification: Terrestrial Wireless Communication/Broadcasting Technologies

\section{References}

[1] S. Haykin, D. J. Tomson, and J. H. Reed, "Spectrum sensing for cognitive radio," Proc. IEEE, vol. 97, no. 5, pp. 849-877, May 2009. DOI:10.1109/ JPROC.2009.2015711

[2] A. V. Dandawate and G. B. Giannakis, "Statistical tests for presence of cyclostationarity," IEEE Trans. Signal Process., vol. 42, no. 9, pp. 2355-2369, Sept. 1994. DOI:10.1109/78.317857

[3] L. P. Goh, Z. Lei, and F. Chin, "Feature detector for DVB-T signal in multipath fading channel," Proc. 2nd Int. Conf. on Cognitive Radio Oriented Wireless Netw. and Commun. (CrownCom 2007), Orlando, FL, USA, pp. 234-240, Aug. 2007. DOI:10.1109/CROWNCOM.2007.4549802

[4] K. Muraoka, M. Ariyoshi, and T. Fujii, "A robust spectrum sensing method based on maximum cyclic autocorrelation selection for dynamic spectrum access," IEICE Trans. Commun., vol. E92-B, pp. 3635-3644, Dec. 2009. DOI:10.1587/transcom.E92.B.3635

[5] G. Huang and J. K. Tugnait, "On cyclostationarity based spectrum sensing under 
uncertain gaussian noise," IEEE Trans. Signal Process., vol. 61, no. 8, pp. 2042-2054, Apr. 2013. DOI:10.1109/TSP.2013.2246158

[6] M. Öner and F. Jondral, "Air interface recognition for a software radio system exploiting cyclostationarity,” Proc. Int'l Symp. IEEE Personal, Indoor, Mobile and Radio Communication (PIMRC 2004), pp. 1947-1951, Sept. 2004. DOI: 10.1109/PIMRC.2004.1368338

[7] A. Papoulis and S. Unnikrishna Pillai, Probability, Random Variables, and Stochastic Processes, Fourth Edition, McGraw-Hill Companies, Inc., 2002.

\section{Introduction}

Recently, cognitive radio techniques have been studied as a means for fulfilling the demands of increasing wireless applications on a limited frequency resource. In cognitive radio networks, spectrum sensing techniques [1] play an important role. Secondary users (SUs) seek a vacancy spectrum for own communication, and if the spectrum is not one used for licensed primary users (PUs), the SUs can use it to communicate. In several types of spectrum sensing techniques, it is well known that a feature detection, which referred to as cyclostationarity detection (CD) $[2,3,4,5]$ using prior knowledge of target signal, has some useful property that is robust to in-band interference signals. In digital communication, actions such as multiplexing and modulating cause cyclostationarity that can be detected by computing a cyclic autocorrelation function (CAF) [6]. However, the computational complexity of $\mathrm{CD}$ based sensing is greater than that of other spectrum sensing techniques, e.g., energy detection based sensing. Although several computationally efficient sensing techniques based on $\mathrm{CD}$ have been presented [3, 4, 5], none of these studies, as far we know, analyzed computation of the CAF itself. In this letter, we present a low computational complexity CAF computation technique for $\mathrm{CD}$ based spectrum sensing in cognitive radio.

\section{CAF of OFDM signals for discrete and finite-time signals}

Let $r(n), v(n)$ and $s(n)$ are received signal, additive white Gaussian noise and PU's orthogonal frequency division multiplexing (OFDM) signal with the number of samples of one OFDM symbol $N_{\mathrm{O}}$, useful data $N_{\mathrm{FFT}}$, and cyclic prefix (CP) $N_{\mathrm{CP}}$. Furthermore, $r(n)=s(n)+v(n)$ and $N_{\mathrm{O}}=N_{\mathrm{FFT}}+N_{\mathrm{CP}}$. An approximated CAF for discrete and finite-time signals is given by

$$
\hat{R}^{\alpha}(v)=\frac{1}{N} \sum_{n=0}^{N-1} r(n) r^{*}(n+v) e^{-j 2 \pi \alpha n \Delta t},
$$

where $N, \alpha, \Delta t, v$, and $\hat{R}^{\alpha}(v)$ are, respectively, the number of samples used to compute the CAF, the cyclic frequency, the sampling period, the lag parameter normalized by $\Delta t$, and the approximated CAF computed by $r(n)$ for $n$ and $N$ samples. We let $\alpha_{k}=k / T_{\mathrm{O}}$ where $k$ and $T_{\mathrm{O}}$ are arbitrary integers and $T_{\mathrm{O}}=N_{\mathrm{O}} \Delta t$, and the CAF of the OFDM signals has peaks at the cyclic frequency $\alpha_{k}$, and $v=T_{\mathrm{FFT}} / \Delta t=N_{\mathrm{FFT}}[6]$. The absolute CAF value, i.e., $\left|\hat{R}^{\alpha}(v)\right|$, is employed for signal detection. 


\section{Presented CAF computation technique}

\subsection{Overview of presented technique}

In a traditional computation as shown in eq. (1), the CAF can be obtained by multiplying the received signal, the delayed received signal and complex periodic function $e^{-j 2 \pi \alpha_{k} n \Delta t}$. In the presented computation technique, setting the sampling frequency of the function as four times (quadruple) the frequency of the function allows the complex periodic function to take the values $\{1+j 0,0-j 1,-1+j 0$, $0+j 1\}$, which significantly reduces the computational complexity. Furthermore, to match the down-sampled complex periodic function, both received signals can be thinned-out to a sampling frequency that is quadruple the arbitrary cyclic frequency, resulting in the generation of some thinned-out signals. Using these thinned-out signals with the complex periodic function, the required number of additions and multiplications needed for CAF computation is approximately halved, which enables computationally efficient $\mathrm{CD}$ based sensing.

\subsection{Details}

We first consider $e^{-j 2 \pi \alpha_{k} n \Delta t}$ for the presented CAF computation technique. Because $\alpha_{k}=k / T_{\mathrm{O}}$, the period of $e^{-j 2 \pi \alpha_{k} n \Delta t}$ is $\left|T_{\mathrm{O}} / k \Delta t\right|=\left|N_{\mathrm{O}} / k\right|$ in general use, and it is seen that the sampling frequency of $e^{-j 2 \pi \alpha_{k} n \Delta t}$ is higher than the cyclic frequency $\alpha_{k}$. The condition in which the sampling frequency of $e^{-j 2 \pi \alpha_{k} n \Delta t}$ is a quadruple of $\alpha_{k}$ can be represented as

$$
\left.e^{-j 2 \pi \alpha_{k} n \Delta t}\right|_{\Delta t=1 / 4\left|\alpha_{k}\right|}=e^{-j 2 \pi k n / 4|k|} .
$$

In order to use $e^{-j 2 \pi k n / 4|k|}$ for the computation, the received signal must be thinnedout so as to match the sampling frequency $1 / \Delta t=4\left|\alpha_{k}\right|$. We let $C^{(g, k)}(l, v)$ denote a signal multiplying $g$ th thinned-out received signal and a delayed received signal with the lag parameter $v$ for the CAF computation with the cyclic frequency $\alpha_{k}$. $C^{(g, k)}(l, v)$ is defined by

$$
C^{(g, k)}(l, v)=r\left(l \frac{N_{\mathrm{O}}}{4|k|}+g\right) r^{*}\left(l \frac{N_{\mathrm{O}}}{4|k|}+g+v\right), \quad g=0, \cdots, \frac{N_{\mathrm{O}}}{4|k|}-1 .
$$

Note that the thinned-out received signal for the computation of $C^{(g, k)}(l, v)$ is obtained not by converting the sampling rate but by literally thinning-out from $r(n)$; thus, an anti-aliasing filter is not used. Needless to say, the received signal is distorted by aliasing. However, it can be considered that the distortion does not significantly affect the signal's cyclostationarity. The cyclostationarity of OFDM signal is caused the parts of $\mathrm{CP}$ and $\mathrm{CP}$ originally [6], to be precise, the repetition pattern which is generated by the $\mathrm{CP}$ and $\mathrm{CP}$ originally. It can be considered that the aliasing distortion of the $\mathrm{CP}$ is very similar to that of the $\mathrm{CP}$ originally because both parts are fundamentally the same. Therefore, it can be considered that the thinned-out operation in the presented technique does not degrade the cyclostationarity of OFDM signal so much. We let $\widehat{Z}^{\alpha_{k}}\left(N_{\mathrm{FFT}}\right)$ denote the CAF computed by the presented technique, and $\widehat{Z}^{\alpha_{k}}\left(N_{\mathrm{FFT}}\right)$ is computed using some thinned-out received signals. Each thinned-out signal can be obtained from $4|k| N / N_{\mathrm{O}}$ samples; therefore, $e^{-j 2 \pi k n / 4|k|}$ can only take the values \pm 1 and 0 , and $\widehat{Z}^{\alpha_{k}}\left(N_{\mathrm{FFT}}\right)$ is defined by 


$$
\begin{aligned}
\widehat{Z}^{\alpha_{k}}\left(N_{\mathrm{FFT}}\right) & =\frac{1}{N} \sum_{g=0}^{\frac{N_{0}}{4|k|}-1} \sum_{l=0}^{\frac{4|k| N}{N_{\mathrm{o}}}-1} C^{(g, k)}\left(l, N_{\mathrm{FFT}}\right) e^{-j 2 \pi l / 4} \\
& =\frac{1}{N} \sum_{g=0}^{\frac{N_{0}}{4|k|}-1} \sum_{l=0}^{\frac{2|k| N}{N_{0}}-1}(-1)^{l} C_{\mathrm{e}}^{(g, k)}\left(l, N_{\mathrm{FFT}}\right)+j(-1)^{l+1} \operatorname{sgn}(k) C_{\mathrm{o}}^{(g, k)}\left(l, N_{\mathrm{FFT}}\right)
\end{aligned}
$$

where $\operatorname{sgn}(\cdot), C_{\mathrm{o}}^{(g, k)}(l, v)$ and $C_{\mathrm{e}}^{(g, k)}(l, v)$ are a sign function, the odd- and evennumbered $C^{(g, k)}(l, v), \quad$ respectively, and $C_{\mathrm{o}}^{(g, k)}(l, v)=C^{(g, k)}(2 l+1, v) \quad$ and $C_{\mathrm{e}}^{(g, k)}(l, v)=C^{(g, k)}(2 l, v)$. In eq. (4), $N_{\mathrm{O}} / 4|k|$ CAFs are computed by each thinned-out received signal, and these CAFs are combined similar to time diversity. Eq. (4) can be further rewritten as

$$
\begin{aligned}
\widehat{Z}^{\alpha_{k}}\left(N_{\mathrm{FFT}}\right)= & \frac{1}{N} \sum_{g=0}^{\frac{N_{0}}{4 k \mid k}-1}\left[\sum_{l=0}^{\frac{|k| N}{N_{\mathrm{o}}}-1}\left\{C_{\mathrm{ee}}^{(g, k)}\left(l, N_{\mathrm{FFT}}\right)-j \operatorname{sgn}(k) C_{\mathrm{oe}}^{(g, k)}\left(l, N_{\mathrm{FFT}}\right)\right\}\right. \\
& \left.-\sum_{l=0}^{\frac{|k| N}{N_{0}}-1}\left\{C_{\mathrm{eo}}^{(g, k)}\left(l, N_{\mathrm{FFT}}\right)-j \operatorname{sgn}(k) C_{\mathrm{oo}}^{(g, k)}\left(l, N_{\mathrm{FFT}}\right)\right\}\right] \\
= & \frac{1}{N} \sum_{g=0}^{\frac{N_{0}}{4 k \mid k}-1}\left[\sum_{l=0}^{\left[\frac{k \mid N}{N_{\mathrm{o}}}-1\right.}\left\{C_{\mathrm{ee}}^{(g, k)}\left(l, N_{\mathrm{FFT}}\right)-C_{\mathrm{eo}}^{(g, k)}\left(l, N_{\mathrm{FFT}}\right)\right\}\right. \\
& \left.-j \operatorname{sgn}(k) \sum_{l=0}^{\frac{|k| N}{N_{\mathrm{o}}}-1}\left\{C_{\mathrm{oe}}^{(g, k)}\left(l, N_{\mathrm{FFT}}\right)-C_{\mathrm{oo}}^{(g, k)}\left(l, N_{\mathrm{FFT}}\right)\right\}\right]
\end{aligned}
$$

where $C_{\mathrm{oo}}^{(g, k)}(l, v), C_{\mathrm{oe}}^{(g, k)}(l, v), C_{\mathrm{eo}}^{(g, k)}(l, v)$, and $C_{\mathrm{ee}}^{(g, k)}(l, v)$ are the odd- and evennumbered $C_{\mathrm{o}}^{(g, k)}(l, v)$ and $C_{\mathrm{e}}^{(g, k)}(l, v)$ for $l$, respectively, and $C_{\mathrm{oo}}^{(g, k)}(l, v)=$ $C_{\mathrm{o}}^{(g, k)}(2 l+1, v), \quad C_{\mathrm{oe}}^{(g, k)}(l, v)=C_{\mathrm{o}}^{(g, k)}(2 l, v), \quad C_{\mathrm{eo}}^{(g, k)}(l, v)=C_{\mathrm{e}}^{(g, k)}(2 l+1, v), \quad$ and $C_{\mathrm{ee}}^{(g, k)}(l, v)=C_{\mathrm{e}}^{(g, k)}(2 l, v)$. Note that these variables are defined to avoid the representation of $(-1)^{l}$ and $(-1)^{l+1}$ as shown in Eqs. (4), (5) and (6). It is seen that eq. (6) includes only the complex addition and bit inverse operations. The computational complexity of eq. (6) is evaluated in the next Section.

\section{Performance analysis}

\subsection{Computational complexity}

Table I. Computational complexity

\begin{tabular}{c|c|c}
\hline Method & $\begin{array}{c}\text { Real } \\
\text { Multiplication }\end{array}$ & $\begin{array}{c}\text { Real } \\
\text { Addition }\end{array}$ \\
\hline$\widehat{Z}^{\alpha_{1}}\left(N_{\mathrm{FFT}}\right)$ computation, eq. (6) & $4 N$ & $4 N-2$ \\
\hline traditional CAF computation, eq. $(1)$ & $8 N$ & $6 N-2$ \\
\hline autocorrelation function $\left(\sum_{n=0}^{N-1} r(n) r^{*}(n+v)\right)$ & $4 N$ & $4 N-2$ \\
\hline signal energy $\left(\sum_{n=0}^{N-1} r(n) r^{*}(n)\right)$ & $2 N$ & $3 N-2$ \\
\hline
\end{tabular}

Table I lists compared the computational complexities of the $\widehat{Z}^{\alpha_{1}}\left(N_{\mathrm{FFT}}\right)$, CAF, autocorrelation function $\left(\sum_{n=0}^{N-1} r(n) r^{*}(n+v)\right)$, and signal energy $\left(\sum_{n=0}^{N-1} r(n) r^{*}(n)\right)$. In the table, complex multiplication is counted as 4 real multiplications and 2 real 


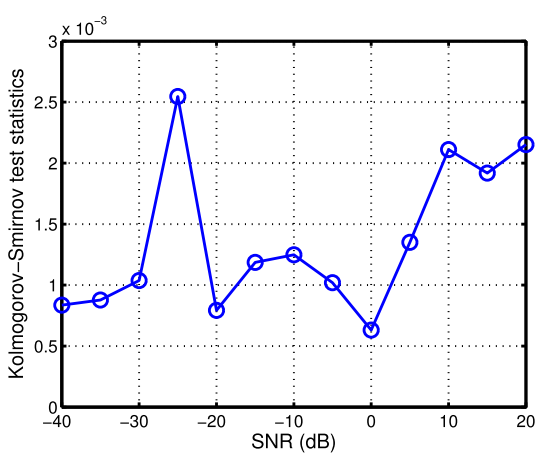

(a) Kolmogorov-Smirnov test statistics of presented techniques for several SNRs.

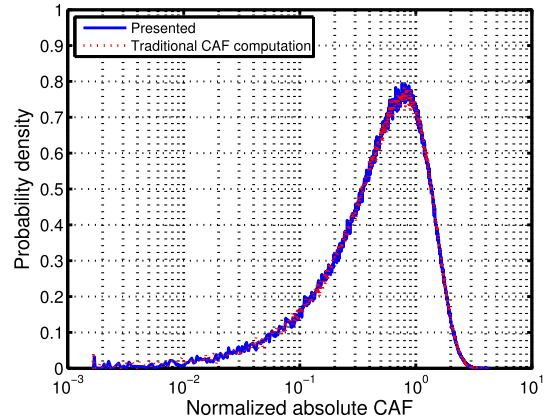

(b) PDFs of presented and traditional CAF computation for $\mathrm{SNR}=-25 \mathrm{~dB}$

Fig. 1. Numerically analyses for statistical property of presented computation technique.

additions, whereas complex addition is counted as 2 real additions. The computation of the presented $\widehat{Z}^{\alpha_{1}}\left(N_{\mathrm{FFT}}\right)$ requires $4 N$ times real multiplication and $4 N-2$ times real addition, and the computational complexity of the presented is the same to that of autocorrelation function. Furthermore, the computational complexity of the presented $\widehat{Z}^{\alpha_{1}}\left(N_{\mathrm{FFT}}\right)$ is less than that of traditional CAF computation although not reach to that of signal energy.

\subsection{Statistical evaluation for absolute CAF}

Fig. 1 shows some numerically statistical properties of the presented technique. Fig. 1a shows the Kolmogorov-Smirnov test [7] between the presented and traditional CAF computation techniques. In Fig. 1a, Kolmogorov-Smirnov test statistics of the received signal at several signal-to-noise ratios (SNRs) are shown for $N_{\mathrm{FFT}}=$ 256 where data symbols were conveyed by 192 subcarriers in these, $N_{\mathrm{CP}}=64$, at $N=10240$ at the single cyclic frequency $\alpha_{1}$. As seen in the figure, the Kolomogorov-Smirnov test values are quite small for all SNRs and can be ignored even though the presented technique does not use an anti-aliasing filter. Fig. 1b shows the probability density functions (PDFs) for the presented and traditional CAF computation techniques at $\mathrm{SNR}=-25 \mathrm{~dB}$, which is the worst case in Fig. 1a. The two PDFs are obtained by normalizing the respective computed absolute CAF using the mean of an absolute traditional CAF. It is seen from the figure that the two functions are closely matched.

\subsection{OFDM signal spectrum sensing performance}

In this subsection, we evaluate the engineering application of the presented technique to the spectrum sensing of OFDM signals. For the evaluation, we employ a simple cylostationarity detection based spectrum sensing technique [3] in which only one peak of the CAF and a threshold are required for signal detection. The performance is evaluated using receiver operation characteristic (ROC) curves. Figs. $2 \mathrm{a}$ and $2 \mathrm{~b}$ shows the ROC curves of $\mathrm{CD}$ based spectrum sensing using the presented and traditional CAF computation techniques for a 1-path Rayleigh fading channel. Both figures show ROC curves for different SNR, $N_{\mathrm{CP}}$ and $N$ at $N_{\mathrm{FFT}}=$ 256 , and the single cyclic frequency $\alpha_{1}$. It is seen that the spectrum sensing 


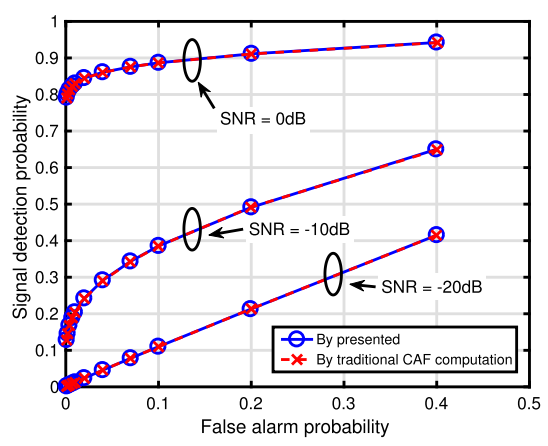

(a) $N_{\mathrm{CP}}=32$ and $N=9216$.

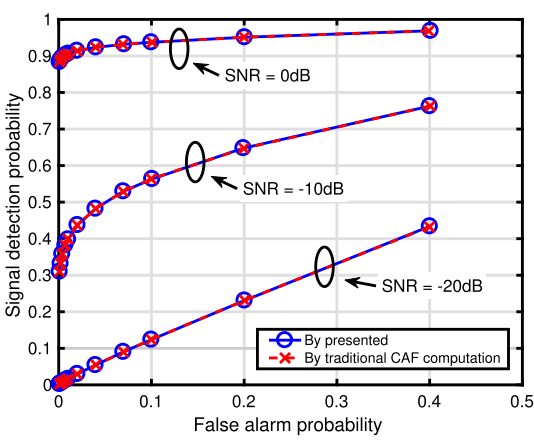

(b) $N_{\mathrm{CP}}=64$ and $N=10240$.

Fig. 2. Numerically analyses for statistical property of presented computation technique.

performance by both techniques are closely matched for all curves. This demonstrates that the presented technique does not affect spectrum sensing performance even though the computational complexity of its CAF is less than that of the traditional technique.

\section{Conclusion}

This letter presented a novel low complexity CAF computation technique for the spectrum sensing of OFDM signals in cognitive radio. The computational complexity of the presented CAF computation technique was less than that of traditional $\mathrm{CAF}$ computation, and furthermore, the complexity of the presented technique was the same to that of autocorrelation function. Numeric analyses of the statistical properties of the presented technique were compared with and found to be approximately identical to those of the traditional CAF computation technique. Further numerical testing verified that the presented does not affect spectrum sensing performance.

\section{Acknowledgement}

This research was supported by the Strategic Information and Communications R\&D Promotion Programme (SCOPE). 